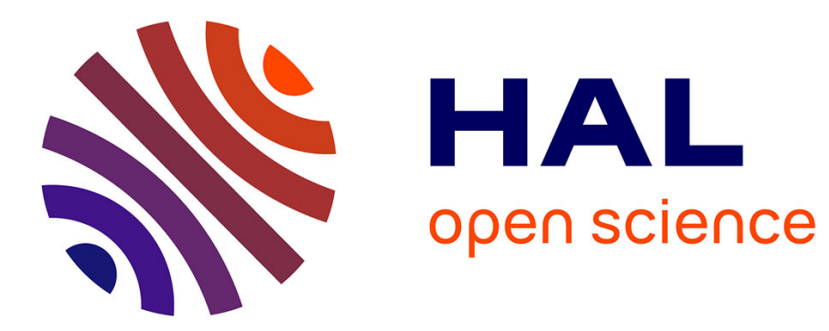

\title{
La coopération franco-britannique en matière de propagande chez l'ennemi (1939-1940)
}

\author{
Maude Williams
}

\section{To cite this version:}

Maude Williams. La coopération franco-britannique en matière de propagande chez l'ennemi (19391940). Relations internationales, 2015, 162, pp.45-62. hal-01740490

\author{
HAL Id: hal-01740490 \\ https://hal.science/hal-01740490
}

Submitted on 23 Mar 2018

HAL is a multi-disciplinary open access archive for the deposit and dissemination of scientific research documents, whether they are published or not. The documents may come from teaching and research institutions in France or abroad, or from public or private research centers.
L'archive ouverte pluridisciplinaire HAL, est destinée au dépôt et à la diffusion de documents scientifiques de niveau recherche, publiés ou non, émanant des établissements d'enseignement et de recherche français ou étrangers, des laboratoires publics ou privés. 


\section{La coopération franco-britannique en matière de propagande chez l'ennemi (1918 - 1940)}

Alliances et coopérations pendant les conflits armés sont des enjeux décisifs pour définir l'issue d'une guerre. Aussi, «des États se rassemblent pour mettre en commun leur sécurité, promouvoir et défendre leurs intérêts communs ${ }^{1}$. À la base de ces alliances, la coopération en matière de stratégie militaire est fondatrice et constitue le thème majeur des négociations construisant la coopération interalliée ${ }^{2}$. En cas de guerre, celle-ci se traduit par une mise en commun du matériel et des hommes, ainsi qu'entre autres, par une coordination au niveau de la stratégie militaire. Ces alliances ne sont cependant pas immunisées face aux confrontations des points de vue divergents des différents membres, bien au contraire ${ }^{3}$. Lors des deux conflits mondiaux, où l'alliance entre la France et la Grande-Bretagne reposait majoritairement sur cet apport mutuel en matière d'hommes et de matériel, nombreuses ont été les fois où les membres des Etats-Majors des deux pays avaient des visions différentes sur les stratégies militaires à employer ${ }^{4}$.

Dans les dernières années de la Première Guerre mondiale un autre secteur de coopération entre les autorités françaises et britanniques émerge : la propagande. Déjà utilisée lors du conflit franco-prussien de 1870/1, la propagande occupe une place grandissante dans le paysage de guerre des années 1914-1918 : les états élaborent des institutions permettant de centraliser la propagande en temps de guerre, d'une part en direction de leur population respective et d'autre part envers l'ennemi et tentent également de mettre au point une propagande commune. ${ }^{5}$ Cette évolution reste néanmoins lente, l'idée d'une coopération n'apparaissant que tardivement dans les discussions interalliées. Bien que la réalisation d'une propagande interalliée soit restée éphémère au cours de la Première Guerre mondiale, on ne peut que constater l'existence d'une coopération franco-britannique en matière de propagande dans les premiers mois du Second conflit mondial.

Afin de comprendre cette évolution, il est nécessaire de se pencher sur la manière dont les autorités, aussi bien militaires que civiles, sont passées d'une coopération exclusivement de stratégie militaire à une coopération plus vaste, englobant cette « nouvelle arme de guerre » de l'époque qu'est la propagande. Aussi, cette évolution soulève de nombreuses questions : Dans quelle mesure la Première Guerre mondiale a-t-elle joué un rôle dans l'élaboration des plans de coopération pendant l'Entre-deux-Guerres ? Existe-t-il des lignes directrices et un schéma clair, élaboré pendant cette période que les autorités françaises et britanniques mettent en application la guerre contre l'Allemagne déclarée ? Quels sont les acteurs impliqués dans les discussions autour d'une propagande commune contre l'Allemagne ? Il est notamment intéressant d'observer si les Alliés ont concrétisé leurs plans d'Entre-deux-Guerres et dans quelle mesure les appartenances nationales ont joué un rôle dans l'élaboration d'une propagande commune à destination de l'ennemi.

\footnotetext{
1 Alexandra de Hoop Scheffer, «Alliances militaires et sécurité collective : contradictions et convergences » dans : Bertrand Badie, Guillaume Devin, Le multilatéralisme, nouvelles formes de l'action internationale, Ed. La Découverte, 2007, p. 57.

2 Max Schiavon, «Les relations entre hauts commandements français et britannique en 1939-1940 », Revue historique des armées, $\mathrm{n}^{\circ}$ 264, 2011, pp. 59-74.

${ }^{3}$ Français et Britanniques dans la drôle de guerre, Actes du colloque franco-britannique tenu à Paris du 8 au 12 décembre 1975, Comité d'histoire de la Seconde Guerre mondiale, Edition du Centre National de la Recherche Scientifique, Paris, 1979.

${ }^{4}$ F. Bédarida, «Convergences et divergences stratégiques franco-britanniques », dans: Actes du colloque franco-britannique tenu à Paris du 8 au 12 décembre 1975, op. cit., pp. 359-379.

5 Olivier Forcade, « Propagande, censure et information en Europe », dans : S. Audouin-Rouzeau, J.J. Becker (dir.), Encyclopédie de la Grande Guerre, Paris, Bayard, 2004, pp. 451-465
} 


\section{LA PREPARATION ET MISE EN PLACE DE LA COLLABORATION}

\section{Les antécédents de la Première Guerre mondiale}

Née lors de la Première Guerre mondiale, l'initiative des Alliés visant à mettre en commun leurs connaissances en matière de propagande et à unir leurs forces pour combattre psychologiquement l'ennemi, peut étonner par sa précocité, étant donné que les institutions de propagande ne bénéficiaient pas d'une longue expérience ${ }^{6}$. L'origine de cette impulsion remonte à une remarque du leader de la propagande italienne, Gallenga Stuart, deux ans après que l'Italie soit entrée en guerre ${ }^{7}$. Cependant, la coopération met du temps à se concrétiser. Alors qu'en juillet 1917 Gallenga fait des propositions aux responsables français et anglais et que la première réunion interalliée ait été prévue pour le mois de mars 1918, ce n'est qu'en août de la même année qu'une rencontre aura réellement lieue. Les causes de ce retard sont grandement liées au processus de formation des institutions nationales de propagande en cours et notamment au remaniement de la Maison de la Presse en France, ainsi qu'aux difficultés à trouver un représentant pour ce comité. ${ }^{8}$

L'objectif des participants ${ }^{9}$ lors de cette rencontre est de «cadrer les «maximes de la politique générale », mais aussi de cibler les types de propagande selon les zones géographiques. [...] $\gg^{10}$. Instaurer une coopération dans le domaine de la propagande signifie néanmoins plus qu'une coopération de stratégie militaire qui a pour but ultime la victoire armée sur l'ennemi. La mise en commun des campagnes de propagande envers l'ennemi pose en effet la question des buts de guerre et oblige les belligérants à s'aligner sur leurs représentations des objectifs de guerre et surtout sur des composantes idéologiques et politiques. $\mathrm{Au}$ cœur des thèmes abordés, ce sont donc des questions politiques, mais également militaires qui sont débattues. En effet, les unes sont liées aux autres, et la propagande doit accompagner la stratégie militaire, comme c'était le cas lors du débat sur l'importance de combattre tout d'abord l'Autriche pour qu'elle sorte de la guerre, ce qui aurait causé alors un réel désavantage pour l'Allemagne. ${ }^{11}$ Après quatre jours de débats, les alliés sortent entendus sur plusieurs points, notamment au sujet de la prolongation de la diffusion d'une propagande antimilitariste et anti-impérialiste contre l'Allemagne. Et bien qu'ils aient également décidé de se réunir de nouveau à Crewe House ${ }^{12}$, la fin de la guerre coupe court à ce projet. Cette rencontre interalliée n'eut pas de conséquence significative sur l'aboutissement de la guerre, mais elle indique clairement que les dirigeants de tous les pays alliés belligérants accordent, à la fin du conflit, une grande importance aux techniques de persuasion.

Ainsi, l'émergence, quoique rapidement interrompue, d'une coopération interalliée dans le domaine de la propagande, marque une prise de conscience importante du rôle que peut jouer l'influence psychologique sur l'ennemi en cas de conflit. Cette initiative ne reste pas sans

\footnotetext{
${ }^{6}$ Concernant les institutions de propagande pendant la Première Guerre mondiale, voir entre autres ; Olivier Forcade, «Propagande, censure et information en Europe», dans : S. Audouin-Rouzeau, J.J. Becker (dir.), Encyclopédie de la Grande Guerre, Paris, Bayard, 2004, pp. 451-465 ; Jean Claude Montant, Jean-Claude Montant, La Propagande extérieure de la France pendant la Première Guerre mondiale : l'exemple de quelques neutres européens, thèse de doctorat d'État, Université Paris 1 - Panthéon Sorbonne, Lille, 1989 ; Michael L. Sanders, Philip M. Taylor, British Propaganda During the First World War: 1914-18, Macmillan Publishers Limited, 1982.

${ }^{7}$ Sanders, Taylor, op. cit., p. 231.

${ }^{8}$ Ibidem.

${ }^{9}$ Entre autres Klobukowski, Northcliffe, Borgese pour l'Italie et Walter Lipmann pour les Etats-Unis.

${ }^{10}$ Georgakakis Didier, La République contre la propagande. Aux origines perdues de la communication d'État en France (1917-1940), Paris, Economica, 2004, p. 106.

${ }^{11}$ Sanders, Taylor, op.cit., p. 232.

${ }^{12}$ Ibidem., p. 233.
} 
lendemain. De chaque côté de la Manche, les autorités militaires, mais aussi civiles continuent à planifier, au regard de leur expérience de la guerre terminée, les possibilités d'organisation, d'institutionnalisation et de collaboration en matière de propagande.

\section{Le Comité franco-britannique de propagande}

Afin de mettre sur pied une coopération franco-britannique dans le domaine de la propagande destinée à l'ennemi, des entretiens préliminaires sont organisés à la demande du gouvernement britannique entre le Comité de Défense Impériale et le Secrétariat Général du Conseil Supérieur de la Défense Nationale. Les premières rencontres attestées ont lieu à Londres les 27 et 28 avril 1939, puis à Paris du 11 au 13 mai $1939^{13}$, et enfin quelques jours aux alentours du 8 juin 1939. Les Britanniques désiraient aborder avec leurs alliés français « la préparation matérielle d'une action de propagande intensive sur les pays ennemis par lancements de tracts et émissions radiophoniques ». ${ }^{14} \mathrm{Il}$ a été décidé le 7 juin 1939 que dans le soucis d'agir d'une manière "particulièrement secrète », un comité restreint serait créé avec Campbell Stuart, chef de la propagande britannique en pays ennemi et l'Amiral Fernet, Secrétaire adjoint du Conseil Supérieur de la Défense nationale pour «étudier les voies et moyens nécessaires » à la réalisation de cette coopération. Ce sous-comité se réunit à partir du lendemain et fournit un rapport très détaillé prévoyant les domaines de coopérations entre les deux alliés en cas de conflit. Les membres du Conseil Supérieur de la Défense nationale ; l'Amiral Fernet, le Général Lahoulle, le Lieutenant-colonel M. Poicignon, le capitaine Fockeu, étaient présents pour représenter la France, et du côté anglais sont intervenus Sir Campbell Stuart, le Lieutenant colonel Brooks, M. Shaw et le capitaine Leathes ${ }^{15}$. Lors de cette rencontre de juin 1939, les représentants militaires des deux pays abordent différents sujets concernant la préparation à la propagande de guerre, notamment en direction de l'Allemagne et de l'Italie. Chacun de ces sujets est ensuite approfondit dans une annexe du «Procès verbal Général des différentes réunions tenues à l'Hôtel des Invalides à Paris le 8 juin 1939 et jours suivants ». On compte alors onze annexes qui abordent de manière détaillée les intentions communes en matière de propagande.

Concernant la question de la propagande par radio, les Français et Britanniques se sont mis d'accord pour échanger des informations sur les essais de «synchronisation de deux stations émettrices travaillant sur la même longueur d'onde moyenne " ${ }^{16}$ afin d'éviter le radio-guidage tout en maintenant les émissions. Quant aux tracts, un échange d'information voit aussi le jour, notamment en ce qui concerne la question des méthodes employées pour «le lancement des tracts à bord des avions », les Britanniques et les Français donnant à la partie tiers le résultat de ces essais. À la suite de ce comité il a été décidé que les aviations britannique et française ferraient de nouveaux essais dans des conditions météorologiques différentes, qu'ils s'en communiqueront les résultats et qu'un accord complet doit être trouvé entre les deux aviations «pour n'avoir qu'un seul système de lancement ». Ensuite, les décisions portent sur les objectifs visés par la propagande. Il en ressort que «le principe de base pour le choix de ces zones d'action était de répandre les tracts d'une manière aussi large que possible au-dessus des zones de densité démographique élevée ». Un autre sujet d'entente était celui des prisonniers de guerre et de leur utilisation comme source d'information. Les

\footnotetext{
${ }^{13}$ National Archives London (désormais NA), FO 898/194 « Note on the meeting held at the Hotel des Invalides on May 11th, 1939 : continuation of the conversations on propaganda in time of war. ».

14 Archives Nationales Paris (désormais AN) F 41/980. Les citations qui suivent sont extraites des procès verbaux des réunions de juin 1939, sauf indication contraire. Ces documents sont également conservés dans les archives nationales de Londres, sous la cote FO 898/194.

${ }^{15}$ Ibidem.

${ }^{16}$ Ibidem.
} 
délégations française et britannique ont sur ce point décidé d'interroger les prisonniers de guerre par le biais d'un questionnaire type et de porter ces informations à la connaissance mutuelles des deux alliés et ce « aussi rapidement que possible». La question de l'impression et du transport des tracts fait également l'objet de discussions et de décisions entre les deux délégations. Il est décidé que les imprimeries pour les tracts et brochures devraient se situer à Lyon, non loin de l'Italie en cas de guerre avec celle-ci et en cas de besoin, dans les zones de Calais - Nancy - Paris pour les actions contre l'Allemagne. Le transport des tracts de l'imprimerie jusqu'aux bases de lancements serait assuré par les Français. Enfin, une alternative à l'envoi des tracts par avion reste l'utilisation de ballons, déjà utilisée lors de la Première Guerre mondiale. Les deux délégations sont en accord sur la nécessité de préparer un stock de ballon en temps de paix et échangent des informations quant à leurs techniques d'élaboration. La proposition des Britanniques de créer des formations spéciales d'aérostiers dépendants de l'Aviation pour lutter contre l'Allemagne est rejetée par les Français qui possèdent déjà des Compagnies régulières d'aérostiers. En conclusion de cette rencontre très fructueuse sur de nombreux points, un poste d'officier de liaison est créé, occupé par le capitaine Leathes qui est mis à la disposition de l'Amiral Fernet et assurera la collaboration entre les deux organismes. La déclaration suivante conclut l'accord de collaboration en matière de propagande entre les Britanniques et Français :

Les Français sont d'accord pour mettre leurs imprimeries à la disposition des Britanniques pour l'impression de leurs tracts de propagande et d'autre part ils assureront par les moyens qu'ils jugeront nécessaires la distribution de ces tracts en Italie. Ils sont aussi d'accord pour coopérer avec les Britanniques pour l'action contre l'Allemagne. En ce qui concerne la radio, la question demande à être étudiée très à fond et dès maintenant il apparaît clairement que la France pourra apporter une aide très importante à la cause des Alliés. Il est admis qu'en cas de guerre le Comité franco-britannique de Propagande étudiera la mise à la disposition des Britanniques des postes d'émissions français, en vue de la propagande dans les pays ennemis ${ }^{17}$.

Au delà d'une simple préparation à la propagande en pays ennemi en temps de guerre, les conclusions de ces rencontres, font ressortir l'intérêt que les deux délégations militaires, largement imprégnées des expériences de la Première Guerre mondiale, accordent à la propagande. $\mathrm{Au}$ mois de juillet, «des débuts de réalisation [sont] obtenus ${ }^{18}$. Les négociations se poursuivent tout au long de l'été $1939^{19}$ et subissent quelques modifications ${ }^{20}$. À travers les rencontres du comité franco-britannique pour la propagande, les représentants militaires des deux nations entendent régler la question des techniques de radiodiffusion, des méthodes générales de coordination et de communication entre les deux alliés, de l'impression et de l'élaboration des tracts ${ }^{21}$.

Il est cependant révélateur de remarquer que ces négociations restent sous le couvert du secret militaire et que les autorités civiles françaises n'en prennent connaissance que tardivement. Le Commissaire Général à l'Information, l'écrivain Jean Giraudoux ${ }^{22}$, en place depuis juillet 1939, n'est informé de ces dispositions que la dernière semaine du mois d'août

\footnotetext{
${ }^{17}$ Ibidem.

18 AN F 41/980. Livraison par les Anglais d'un Mémorandum le 6 juillet 1939 sur des suggestions de propagande vers l'Allemagne.

${ }^{19}$ FO 898/194. Les comptes rendus anglais font mention des rencontres suivantes : 28-29 juillet, 17 août, 13 août, 24 août.

${ }^{20}$ NA FO 898/194. On peut citer l'emplacement des imprimeries prévues pour la production des tracts qui, en aout est modifié et défini Reims et Grenoble comme les nouveaux lieux d'impression.

${ }^{21}$ London, FO 898/194 «Anglo-french propaganda Council : Note of the meeting held at the Hotel des Invalides Paris, on Thursday, August 17, 1939, and following day. ».

${ }^{22}$ Sur Jean Giraudoux en tant que Commissaire général à l'Information voir l'article de Didier Georgakakis, « Le Commissariat Général à l'Information et la drôle de guerre », Mélanges de l'Ecole française de Rome. Italie et Méditerranée, vol. 108, 1996, et l'ouvrage précédemment cité du même auteur.
} 
alors que les tensions avec l'Allemagne se font de plus en plus ressentir ${ }^{23}$. Ce n'est donc qu'une fois les négociations terminées que Giraudoux est averti d'une collaboration avec la Grande-Bretagne, et qu'il reçoit l'ordre de se mettre en contact étroit avec ses futurs collaborateurs anglais ${ }^{24}$. Le mercredi 30 août 1939, Sir Campbell Stuart se rend à Paris afin d'y rencontrer les autorités françaises et de préparer la mise sur pied d'une coopération franco-anglaise en matière de propagande vers l'ennemi si la guerre devait être déclarée ${ }^{25}$. À cette occasion, il rencontre tout d'abord l'amiral Fernet, puis M. Giraudoux. Des réunions extraordinaires concernant la collaboration dans le domaine des émissions radiophoniques sont organisées les 31 aout et $1^{\text {er }}$ septembre 1939. De toutes ces rencontres s'en suit la création d'un comité franco-britannique - civil cette fois -, dont les travaux «doivent progressivement entrer dans l'activité de la IIIème Division (Section de propagande en pays ennemi) du Commissariat. Celui-ci est cependant composé d'agents de liaison disposant d'un statut militaire important tel le Capitaine de Vaisseau Le Comte mis en relation avec le Colonel Sinclair, officier de liaison britannique attaché en Angleterre à la section de Sir Campbell Stuart, ainsi que le vicomte Strathallan ${ }^{26}$. Afin de renforcer la «conception intellectuelle » de la propagande commune, un «représentant de l' 'editorial staff' ${ }^{27}$ anglais, M. Noel Coward est envoyé à Paris, remplaçant le capitaine Leathes. De son côté Giraudoux nomme André Maurois pour «contrôler les rapports de la liaison Britannique avec les membres de la Section française de Propagande en Pays ennemi ${ }^{28}$.

Prenant racine à la fin de la Première Guerre mondiale et se développant pendant l'Entre-deux-Guerres, les bases d'une coopération franco-britannique en matière de propagande sont déjà présentes lors du déclenchement des hostilités contre l'Allemagne. Ces dispositions, issues des cercles militaires, s'élargissent aux autorités civiles et permettent l'instauration d'un dialogue régulier entre les deux alliés.

\section{La mise en place d'un dialogue régulier en temps de guerre}

La première réussite est effectivement tout d'abord celle du dialogue. Ininterrompu pendant l'Entre-deux-Guerres, il se poursuit et s'intensifie une fois la guerre déclarée. Concrètement, à la mi-septembre, les autorités alliées responsables de l'envoi des tracts entrent en contact : le Lieutenant Colonel R.A.D. Brook se rend à Paris trois jours, du $17 \mathrm{au}$ 19 septembre afin de rencontrer ses homologues français. Dans son rapport, il mentionne ses rencontres avec les différentes autorités françaises concernées, parmi lesquels on peut citer le Capitaine de Vaisseau Le Comte et le Capitaine Schuhl, et participe à la réunion du comité franco-britannique pour la propagande en pays ennemi. Anglais et Français se réunissent toutes les semaines à l'Hôtel Continental (siège du Commissariat Général à l'Information), afin d'élaborer une ligne commune de propagande et faire le bilan des actions entreprises de part et d'autre. On constate, dans la composition de ces comités, qu'un certain équilibre s'est installé dans la représentation des autorités militaires et civiles des deux délégations. Lors de ces réunions hebdomadaires sont présents de façon - presque - permanente M. de Saint Jean, le capitaine le Vicomte Strathallan, Peter Pitt-Millward, le Capitaine de Vaisseau Le Comte, M. Tonnelat, M. Vermeil, Noel Coward, M. Donati, et dix-neuf autres personnes y assistent occasionnellement. Les sujets abordés sont variés et portent aussi bien sur le contenu de la propagande, que sur son efficacité, ou bien encore sur ses techniques. Il s'agit principalement

\footnotetext{
${ }^{23}$ AN F 41/980.

${ }^{24}$ NA FO 898/194, « Note of the visit by Sir Campbell Stuart to Pris August 30th - September 1st, 1939 ».

${ }^{25}$ NA FO 898/194, « Note of a visit by Sir Campbell to Paris ».

${ }^{26}$ Ibidem.

${ }^{27}$ AN F 41/980.

${ }^{28}$ Ibidem.
} 
d'échanger des documents ${ }^{29}$, de faire le bilan des actions menées en commun et d'en projeter de nouvelles.

Les échanges de renseignements sur l'ennemi constituent une grande partie de la coopération franco-britannique. Lors des rencontres, il est souvent question des rapports des interrogatoires de prisonniers de guerre allemands dont les questionnaires avaient été préparés pendant l'Entre-deux-Guerres. L'utilisation des prisonniers comme source de renseignements est en effet une pratique précieuse pour «rechercher les effets produits par [notre] propagande $»^{30}$, s'informer de son efficacité, ainsi que sonder le moral des soldats et de la population ennemie ${ }^{31}$. Ces informations permettent ensuite aux alliés d'orienter leur propagande en fonction des angoisses et des attentes de la cible visée. Elles viennent compléter l'éventail des questions déjà posées par les autorités militaires telles que «La guerre est-elle populaire ?, Y a-t-il du sabotage ?, Combien ? ${ }^{32}$. Celles rédigées pour les institutions de propagande portent surtout sur les tracts ennemis et questionnent l'impact des arguments, par le biais de ces questions : " Avez vous vu, avez vous lu des tracts provenant de l'ennemi ?, Qu'en pensez vous ?, Qu'en pensent vos camarades ? Quelle importance leur reconnaît-t-on? Croit-on aux textes de ces tracts ? ${ }^{33}$. Elles évoquent également la manière dont ils ont pris connaissance de ces tracts en demandant : "Avez vous été témoin de leur chute ? Ou bien vous ont-ils été remis secrètement? $\gg{ }^{34}$. Les réponses à ces interrogations apportent des informations sur le moral ainsi que sur l'influence ou l'absence d'influence de la propagande alliée. Ainsi, un résumé d'interrogatoire français de prisonniers de guerre allemands, daté du 9 janvier 1940, indique que certains arguments de propagande n'ont pas trouvé d'écho dans l'armée allemande ${ }^{35}$. Il en va de même pour la campagne de déstabilisation de l'armée allemande par le blocus franco-britannique ${ }^{36}$, mais également de la question de la responsabilité de la guerre. En effet, les autorités militaires françaises déplorent

En ce qui concerne la question primordiale de la responsabilité de la guerre, tous les prisonniers interrogés se sont déclarés convaincus de la sincérité des intentions pacifiques du Führer à l'égard de la France ; ils ne voient manifestement aucun rapport entre l'agression allemande contre la Pologne et les déclarations de guerre de l'Angleterre et de la France $[\ldots]^{37}$.

Les autorités britanniques sont d'avis que les soldats allemands continueront à ne pas croire aux nouvelles alliées jusqu'à ce qu'ils les « lisent imprimées dans un journal allemand $\gg^{38}$. En outre, les informations obtenues influencent de manière non négligeable les orientations de la propagande française et anglaise. Lors d'une réunion hebdomadaire du

\footnotetext{
${ }^{29}$ Ibidem. Les documents transmis le 16 janvier 1940 sont représentatifs du type d'informations échangées lors de ces réunions. Il s'agit entre autre d'un rapport de la BBC «Weekly Monitoring report », d'un rapport sur les prisonniers de guerre, $\mathrm{d}$ 'une brochure anglaise ainsi que des tracts.

30 AN F 41/980

${ }^{31}$ Sur les interrogatoires de prisonniers de guerre pendant la Seconde Guerre mondiale, voir les ouvrages ; Sönke Neitzel Harald Welzer, Soldaten: Protokolle vom Kämpfen, Töten und Sterben, Frankfurt am Main, 2011 ; Sönke Neitzel, Abgehört :Deutsche Generäle in britischer Kriegsgefangenschaft 1942-1945, List Taschenbuch, Berlin, 2007.

32 AN F 41/980

${ }^{33}$ Ibidem.

${ }^{34}$ Ibidem.

35 Service Historique de la Défense, 27N67, « Propagande contre l'Allemagne - Note pour le Commissariat Général à l'Information (Commandant Schiffer) », 9.01.1940.

${ }^{36}$ Gaël Birotheau, Mythe ou réalité d'un blocus maritime réussi : politiques et moyens mis en oeuvre par la marine française (Septembre 1939 - Juin 1940), thèse sous la direction de Vincent Joly, Université Rennes 2 , 2011.

${ }^{37}$ Ibidem.

${ }^{38}$ Ibidem.
} 
comité franco-anglais, il est par exemple mentionné que «le moment était venu de commencer notre propagande même sur les troupes » car "l'impression générale tirée de l'interrogation des prisonniers était que des signes de lassitudes étaient évidents ${ }^{39}$. Les exemples de cet acabit sont nombreux et constituent une partie importante des renseignements utilisés pour fonder la propagande alliée, en parallèle avec les rapports sur le moral et la situation en Allemagne fourni par d'autres institutions ${ }^{40}$.

Ces documents proviennent pour la plupart de sources tels que l'Institut des Etudes Européennes du côté français ou bien de l'Electra House du côté anglais ${ }^{41}$. Ces rapports contiennent notamment des revues de presse des journaux allemands, des informations sur le moral de la population et des soldats, obtenues parfois par quelques informateurs secrets stationnés en Allemagne ${ }^{42}$. Les documents sont échangés lors de ces réunions ainsi qu'entre les différentes institutions en relation. Ainsi, le Commissariat Général à l'Information reçoit de manière régulière les rapports d'Electra House. Ceux-ci consistent en plusieurs rapports sur l'Allemagne mais également la Russie ou les pays annexés. En ce qui concerne l'Allemagne, les services anglais analysent les discours de la presse et de la radio allemande. Ils donnent également une étude de la situation interne du pays en se basant notamment sur les médias allemands. De la part des autorités françaises, les Anglais reçoivent les «bulletins hebdomadaires d'information »du Commissariat Général à l'Information ${ }^{43}$. Ceux-ci sont divisés en trois parties ; la première concerne "les thèmes de la propagande étrangère ${ }^{44}$, la seconde «les causes de discussion ou d'inquiétude dans l'opinion publique ${ }^{45}$ et la troisième annonce «quelques sujets à traiter $»^{46}$, ce qu'il faut comprendre comme les sujets à diffuser dans les médias. On constate alors une réciprocité effective dans l'échange des renseignements entre Anglais et Français. Ces échanges sont très suivis lors de la « drôle de guerre » et sont complétés par d'autres types de renseignements et de documents, plus techniques.

En effet, en dehors de leurs réunions, les institutions prennent contact et échangent des rapports d'ingénieries. Les Anglais transmettent par exemple un rapport concernant le ballon lance-tract de Type « $\mathrm{M}$ » et les Français font parvenir à leur allié celui du « lance-tract en caoutchouc Bognier Burnet $\gg^{47}$. Ils échangent également des informations sur les ballons allemands tombants en France. Après que les ingénieurs français ont analysé le ballon ennemi, ils envoient un rapport et le ballon aux autorités britanniques ${ }^{48}$. Le dialogue entre les deux nations est très intense et s'actualise toutes les semaines grâce aux rencontres hebdomadaires du comité franco-britannique pour la propagande. Ces réunions représentent aussi l'occasion de dresser un bilan des tracts envoyés, des émissions diffusées en langue allemande et de mettre en commun ses projets de propagande contre l'ennemi. À chaque fin de réunion, Lord Strathallan communique le détail des distributions de tract pour la semaine

\footnotetext{
${ }^{39}$ AN F 41/ 980.

40 On peut citer comme institution de renseignement l'Institut des Etudes Européenne de Strasbourg qui fournit quantité d'informations aux autorités militaires et civiles françaises sur la situation de la population allemande et sur les campagnes de propagande de l'ennemi. (Archives départementales du Bas-Rhin, 98 AL 649-659)

${ }^{41}$ Les documents des deux institutions se retrouvent dans les archives nationales et militaires françaises et dans les archives nationales consultées à Londres.( AN F 41/ 980 et NA FO 371/24312-18)

42 C'est le cas pour «Neu Beginnen », un groupe d'intellectuels marxistes qui ont formés une union de socialistes allemand au Royaume Uni et un mouvement clandestin anti-nazi en Allemagne.

${ }^{43}$ NA FO 371/24312-18.

${ }^{44}$ NA FO $371 / 24316$.

${ }^{45}$ Ibidem.

${ }^{46}$ Ibidem.

${ }^{47}$ AN F/41/991, «'M' Baloon Manual » et « Rapport d'essais concernant les ballons porte tracts en caoutchouc Bognier Burnet », transmit le 17.11.1939 au Commissariat pour le « remettre à M. Noël Coward, président de la mission britannique de propagande en pays ennemi ».

${ }^{48}$ Ibidem. Correspondance entre le commissaire Général de l'information et le Ministre de l'Air, 31.1.1940.
} 
écoulée. Ainsi, on apprend par exemple que du 2 au 25 mars 1940, ce sont plus de 1714000 tracts de 17 sortes différentes qui ont été largués sur plus de 18 villes allemandes par les Anglais, ainsi que plus d'un millions jetés par les Français du $1^{\mathrm{er}}$ au 14 mars $^{49}$. Concernant les émissions radiophoniques, les bilans sont aussi échangés, même si on remarque un déséquilibre. En effet, alors que les Anglais transmettent aux Français les scripts de leurs émissions en langue allemande diffusées par la BBC, les scripts français se font attendre. Le 22 septembre 1939, les Alliés anglais demandent la réciprocité ${ }^{50}$, mais aucune source n'a pu être trouvée pour confirmer que l'échange eut lieu par la suite, bien que les thèmes à évoquer dans les émissions en langue allemande fassent partie des débats des réunions hebdomadaires ${ }^{51}$.

Les autorités françaises et britanniques ont su, rapidement après le début des hostilités, se mettre en contact et échanger un nombre important d'informations et de renseignements capitaux pour la réalisation d'une propagande susceptible d'avoir une influence auprès de l'ennemi. La diversité et la régularité des informations échangées indiquent qu'une évolution par rapport à la Première Guerre mondiale a eu lieu : la propagande est prise au sérieux, institutionnalisée et fait l'objet d'une réelle coopération interalliée. Mais qu'en est-il des projets de création d'une propagande commune qui avaient émergés pendant l'Entre-deuxGuerres ? La coopération franco-britannique dépasse t-elle le simple cadre des échanges de renseignements?

\section{L’ALIGNEMENT DES PROPAGANDES ALLIEES}

\section{Les actes de propagande et les lignes directrices}

Sur certains points, la coopération prend pendant la drôle de guerre, une forme plus pratique. Elle se concrétise dans les faits à la fois à travers une collaboration très étroite dans l'élaboration d'une ligne directrice de propagande contre l'ennemi, mais également par la confection de tracts et d'émissions radiophoniques en langue allemande.

Le 5 septembre 1939 apparaît dans une note pour le commissaire Général à l'Information venue du Service de Propagande chez l'ennemi (SPE) l'idée qu' «il serait fort opportun d'instituer une liaison parallèle avec les services anglais pour tout ce qui concerne la rédaction de ces tracts et l'esprit général dans lequel ils sont conçus. ${ }^{52}$. Cette tâche s'ajouterait à celle dirigée par Monsieur Comert d'échanger de la documentation avec les alliés. Le 16 septembre 1939, le premier résultat d'un échange de tract se concrétise. Les trois tracts anglais envoyés aux autorités françaises pour une étude reçoivent une critique sévère. Les rapports des Alsaciens qui ont examiné les tracts anglais font remarquer non seulement des erreurs de langue, mais aussi de forme : ils critiquent notamment la langue et le choix des mots ${ }^{53}$, mais également le style qui paraît à leurs yeux «recherché et ridicule $»^{54}$. Enfin, les Français proposent aux Anglais de faire, comme eux, appel à des réfugiés allemands pour rédiger leurs tracts et concluent par une « leçon de propagande »:

\footnotetext{
${ }^{49}$ AN F 41/980, « distribution of leaflets - March $1940 »$.

${ }^{50}$ Ibidem., « Note pour Foucques Du Parc, Quai D’Orsay, Radio », 22 septembre 1939.

${ }^{51}$ Ibidem.

${ }^{52}$ AN F 41/980 « Note pour le Commissaire général », 5 septembre 1939.

${ }^{53}$ AN F 41/980 « Note à propos des trois tracts communiqués par la mission anglaise », 18 septembre 1939, et «Observations concernant les tracts anglais », 16 septembre 1939, «L'expression «mit kühl erwogenem Vorsatz » et l'expression «wir hegen keine Feindseligkeit » ne sont pas allemandes ...».

${ }^{54}$ Ibidem.
} 


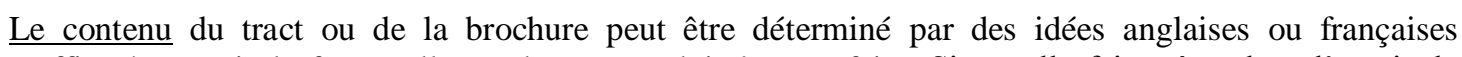
nettement affirmées. Mais la forme allemande peut et doit être parfaite. Sinon elle fait naître dans l'esprit du lecteur allemand le scepticisme ou la moquerie, affaiblissant d'autant l'effet produit ${ }^{55}$.

Ces échanges d'expertise se renforcent au fil des mois et deviennent des rencontres hebdomadaires. En effet, il est régulièrement fait mention des tracts échangés et des avis des uns et des autres. C'est ainsi qu'à travers les procès verbaux de ces réunions on voit apparaître les lignes directrices de la propagande française et anglaise qui forment une propagande alliée allant dans la même direction et abordant les mêmes sujets. Ces échanges ne se limitent pas aux tracts mais concernent également la propagande par haut-parleurs au front ${ }^{56}$, ainsi que les émissions radiophoniques, notamment celles de la $\mathrm{BBC}^{57}$.

Parmi les nombreux thèmes de propagande, deux occupent une place importante et récurrente dans les tracts et les discours français et britannique envers l'Allemagne. L'objectif principal qui ressort à la fois des réunions, mais également de l'analyse empirique des tracts ${ }^{58}$ et des émissions de radios alliées ${ }^{59}$, est la mise en confrontation entre le peuple allemand et ses dirigeants, membres du parti national-socialiste. Sur cette tentative de soulever le peuple contre ses dirigeants, M. Coward fait remarquer le 19 janvier 1940 que les deux tracts qu'il a reçu des autorités françaises font «tous les deux appel à la révolution ${ }^{60}$ et que «cette tendance $»^{61}$ semblait clairement être le fruit d'une politique bien déterminée, ce à quoi son collègue français $\mathrm{M}$. Donati répond que « ceci avait été dès le début le leitmotiv préféré par le GQG $»^{62}$. Du côté anglais, leurs tracts parlent d'eux-mêmes : le tract diffusé en novembre 1939 et portant le titre «Der Führer spricht» (le Führer parle) met en avant la perfidie d'Hitler par le biais de citations issues de ses discours d'avant-guerre. La citation de 1933, extraite, selon le tract, d'une interview entre Hitler et un journaliste anglais est la suivante :

Croyez vous que nous éduquions notre jeunesse - qui représente notre avenir et à laquelle tous nous tenons -, pour la laisser combattre sur le champ de bataille ?... Personne de nous ne pense à déclarer une guerre à la Pologne à cause du corridor (V.B. 20. Octobre 1933) ${ }^{63}$.

Elle a pour but d'inciter le peuple à se révolter contre Hitler ou du moins à réfléchir sur le sens de la guerre, qui selon la propagande alliée, est voulue par le Führer. Tous les arguments sont utilisés pour montrer l'absurdité de la politique de celui-ci; des restrictions alimentaires jusqu'à la dénonciation des membres du parti nazi, en passant par la critique du pacte germano-soviétique ${ }^{64}$. Le second argument majeur de la propagande franco-anglaise consiste à renforcer, auprès des Allemands, l'idée d'une alliance inébranlable entre la France et la Grande-Bretagne et ainsi contrecarrer l'argument majeur de la propagande nationale-

\footnotetext{
55 Ibidem.

${ }^{56}$ AN F 41/980 « réunion du 4 avril 1940 ».

${ }^{57}$ AN F 41/980 «B.B.C. Broadcasts in German ».

${ }^{58}$ Kirchner Klaus, Fluglblätter im Zweiten Weltkrieg, t. 2, Flugblätter aus Frankreich 1939-1940, t.3 Flugblätter aus England, D+C Verlag, Erlangen, 1982.

${ }^{59}$ Diverses sources telles que les fonds INF 1/161 et INF1/172 renseignent sur le contenu des émissions de radio de la BBC pour l'Allemagne et les rapports allemands des archives militaires de Fribourg en Brisgau (fond RW4/242) renseignent sur le contenu des émissions, à la fois françaises et anglaises.

${ }^{60}$ AN F 41/980 « réunion du 29 février $1940 »$.

${ }^{61}$ Ibidem.

${ }^{62}$ Ibidem.

${ }^{63}$ Traduction de l'auteur. Kirchner Klaus, Flugblätter psychologische Kriegsführung im Zweiten Weltkrieg in Europa, Carl Hanser Verlag, München, 1974, p. 45.

${ }^{64}$ Sur ces différents arguments, voir notamment les tracts rassemblés dans les ouvrages de Klaus Kirchner, op. cit.
} 
socialiste ${ }^{65}$. La réalisation du «tract commun » peut être pris comme exemple pour montrer à la fois la coopération concernant l'élaboration d'un tract, ainsi que pour illustrer la thématique de propagande alliée qu'est la forte union franco-britannique.

La réalisation du tract «Darum Krieg » constitue le premier projet de tract commun franco-britannique. Après être passé par les mains des autorités françaises et anglaises, chacun ayant ajouté sa part de photographie, de photomontage et de citation, le tract est soumis une nouvelle fois pour être validé. Il est ensuite livré aux imprimeurs français et enfin déversé en Allemagne par la Royal Air Force et l'Armée de l'Air française de fin février à début mai 1940 sur les villes de Merzig, Karlsruhe et de Munich ${ }^{66}$. Ce tract se présente sous forme de dépliant mêlant texte, image (photomontage) et schéma. Il s'agit plus particulièrement de représenter le contingent armé français et anglais dans tous ses domaines (aviation, marine, infanterie) afin de montrer la supériorité à la fois économique, mais aussi numéraire des troupes alliées. De plus, la volonté de prouver l'unité inébranlable de l'Alliance francobritannique ${ }^{67}$ sert également l'objectif de ce tract qui consiste à faire douter les Allemands d'une issue victorieuse de la guerre pour l'Allemagne. Cependant, les démarches de réalisation sont extrêmement longues, particulièrement en matière de propagande qui doit être rapide et adaptée aux évènements d'actualités. Le projet avait en effet était lancé le 17 novembre 1939, mais ce n'est que le 16 janvier 1940 que la version définitive est validée et il n'atteint la population allemande qu'à partir de février 1940.

Parallèlement au choix de thématiques communes, la définition de la forme et les techniques de propagande font également partie de la coopération. Les Alliés s'influencent mutuellement; les Anglais décident, après avoir reçu plusieurs remarques sur le manque d'illustration, «d'adopter des dessins satiriques ${ }^{68}$ et de créer des slogans. La période d'enclenchement d'une thématique de propagande n'est également pas laissée au hasard, renforçant ainsi le caractère réfléchi d'une propagande franco-britannique à l'unisson. Le 13 novembre 1939, le GQG ordonne «d'aller de l'avant à une grande échelle en matière de propagande ${ }^{69}$. L'origine de cet ordre se trouve dans la lecture de rapports « obtenus par des agents durant les trois dernières semaines », c'est à dire fin octobre-début novembre 1939. Ces rapports «pointent tous le fait que l'opinion en Allemagne est sérieusement en colère ${ }^{70}$. Il s'agt alors pour l'Etat Major d'utiliser cette information pour intensifier la propagande en relation avec les raisons de cette colère, c'est-à-dire les conditions de vie difficiles des Allemands. Les tracts doivent donc évoquer des thématiques qui tournent autour des problèmes quotidiens tels que l'inflation et le manque de nourriture ${ }^{71}$. De plus, au fil des mois, la propagande franco-britannique se veut de plus en plus régionale : les alliés établissent des émissions en dialecte pour «fournir une couleur locale (à nos) émissions en langue allemande $\gg^{72}$ et ils envoient des tracts visant une cible bien définie comme par exemple les paysans du Bade Wurtemberg ${ }^{73}$. En s'adressant directement à un public spécifique, ils espèrent ainsi rendre leur propagande plus authentique et plus persuasive.

\footnotetext{
${ }^{65}$ Fagot Maude, « La guerre des ondes entre la France et l'Allemagne pendant la "drôle de guerre" », Revue Historique (671), mars 2014, pp. 643-644.

${ }^{66}$ Kirchner Klaus, op.cit., p. 176.

${ }^{67}$ Kirchner Klaus, op.cit., pp. 62-63.

${ }^{68}$ AN F 41/980 «Réunion du 14 mars 1940 ».

${ }^{69}$ AN F 41/980 « Meeting of November 13, 1939 ».

70 Traduction de l'auteur. Idem.

${ }^{71}$ Ibidem. Un autre exemple aurait pu être cité, celui de la présence nouvelle de SS et SA au front qui fait stopper la critique de leur absence au combat, jusqu'alors très fortement exploitée. « Réunion du $1^{\text {er }}$ février 1940 ».

${ }^{72}$ AN F 41/980 « Réunion du 4 avril $1940 »$.

${ }^{73}$ Bibliothèque Documentaire Internationale Contemporaine Z 0005, Tract intitulé « Deutsche Bauern aus Baden und Württemberg » qui dénonce le plan déplacement des Badois vers les territoires de l'Est et notamment de la Pologne.
} 
Ces efforts franco-britanniques pour homogénéiser leurs propagandes n'est pas passée inaperçue par les autorités militaires allemandes. En effet, celles-ci remarquent dans leurs rapports sur la propagande ennemie qu'il «n'y a plus de différence désormais entre la radio française et anglaise. Les deux voix coïncident parfaitement dans la méthode et le ton ${ }^{74}$ et ce dès la mi-octobre 1939. De plus, un mois plus tard, le même constat est fait :

Les propagandes radiophoniques françaises et anglaises sont à présent dans leur tactique et leur ton tellement similaires, que les émissions de Strasbourg et de Daventry coïncident presque parfaitement dans leur forme, contenu, et construction ${ }^{75}$.

Il semble ainsi aux autorités allemandes que les deux alliés travaillent « main dans la main pour la combinaison de leurs affirmations et accusations $\gg^{76}$. Ces témoignages des autorités allemandes reflètent la certaine efficacité de la coopération franco-britannique en matière de propagande. Néanmoins, même si la coopération est une réussite dans les aspects que nous venons d'évoquer (échange d'informations, dialogue et coordination des thématiques de propagande), quelques ombres viennent obscurcir le tableau.

\section{Les limites et problèmes de la coopération}

Bien que la coopération dans le domaine de l'échange d'information semble fonctionner et porter ses fruits, elle se heurte en effet à certaines limites. Apparaissent tout d'abord des obstacles relevant du domaine de la confiance entre les alliés. D'une part, les autorités françaises gardent le silence autour de leurs postes clandestins infiltrés en Allemagne et en Autriche, les «postes de la liberté »: deutscher Freiheitssender et österreichischer Freiheitssender. Les émissions provenant desdites « radio noires » font croire aux auditeurs allemands et autrichiens qu'elles sont diffusées de leur propre pays par des groupes socialistes et communistes, alors qu'elles sont en réalité le fruit d'une propagande radiophonique française ${ }^{77}$. Cependant, lorsque les autorités anglaises demandent à maintes reprises des informations sur ces émissions ${ }^{78}$, la réponse française laisse place au silence. En affirmant ne rien savoir ${ }^{79}, \mathrm{M}$. Vermeil, dont l'ignorance à ce propos est sujet à caution puisqu'il fait partie des collaborateurs pour les émissions françaises en langue allemande, révèle une des limites de la coopération franco-anglaise ${ }^{80}$. En outre, ce seuil de confiance est exprimé explicitement dans un document du 16 janvier 1940 :

D'après les instructions du M. le Colonel Hazard, la documentation en question qui sera centralisée par M. Robert de Saint Jean qui assistera d'autre part aux réunions du samedi pour y présenter le comité anglais. Avant de remettre n'importe quelle pièce aux Anglais, il devra l'examiner attentivement, et retenir tout ce qu'il n'est pas désirable de communiquer à nos Alliés pour telle ou telle raison ${ }^{81}$.

Ainsi, toutes les informations françaises ne sont pas transmises aux alliés britanniques mais sont filtrées. L'échange d'informations se heurte donc à des barrières nationales et n'est pas illimité, du moins de façon évidente de la part des autorités françaises.

\footnotetext{
${ }^{74}$ BArch-MA, RW4/242, « Lagebericht der Rundfunk », 16.10.1939.

75 BArch-MA, RW4/241, 7.11.1939.

${ }^{76}$ Ibidem.

${ }^{77}$ Pütter Conrad, Rundfunk gegen das «Dritte Reich», Deutsche Rundfunkaktivitäten im Exil 1933-1945- ein Handbuch, Munich, K.G.Saur, 1986.

${ }^{78}$ Evoqués a plusieurs reprises dans les réunions hebdomadaires: 16 janvier 1940, 25 janvier 1940, $1^{\text {er }}$ février

${ }^{79}$ AN F 41/980, « Réunion du 16 janvier 1940 ».

${ }^{80}$ Bien que le doute puisse subsister quant à la réelle connaissance de M. Vermeil sur l'existence de ces émissions, il est tout de même approuvé qu'elles sont d'origines françaises. Le manque de communication et la volonté de garder ses émissions secrètes est clairement visible.

${ }^{81}$ AN F 41/980 Réunion du 16 janvier 1940.
} 
Un autre domaine, celui de la collaboration matérielle, se met également en place dès les premiers jours du conflit mais s'avère être moins efficace que prévu lors des réunions organisées pendant l'Entre-deux-Guerres. Les intentions initiales étaient multiples : brouiller les émissions ennemies, préparer les stocks de ballonnets pour lancer les tracts, organiser en commun leur impression et leur distribution ${ }^{82}$. Le rapport du colonel Schiffer sur la visite de Sir Campbell Stuart au mois d'avril 1940 met en lumière les difficultés rencontrées dans le domaine du brouillage des émissions ennemies :

Il est incontestable qu'en cette matière, une coordination complète a été obtenue du coté britannique, entre les mains de Sir Campbell Stuart, alors qu'il n'est pas de même du côté français où coexistent plusieurs organes, sans liaisons effectives entre eux et sans intervention d'une autorité coordinatrice ${ }^{83}$.

Le retard dans ce domaine résulte, selon l'analyse du colonel, du manque de coordination de la part des autorités françaises ; un problème récurrent dans l'appareil de propagande national ${ }^{84}$. De surcroît, les autorités françaises affirment qu'il est « techniquement onéreux et difficile de brouiller efficacement une grande surface $»^{85}$.

Dans le domaine des tracts, qui constitue le deuxième principal moyen de diffusion de la propagande, les alliés se voient confrontés à d'autres problèmes. Au mois de septembre 1939, sous l'impulsion anglaise, l'organisation de l'impression et de la distribution commune des tracts se met en marche. Les autorités britanniques proposent :

$1^{\circ}$-Tous les tracts qu'ils aient été rédigés à Londres ou à Paris, seront imprimés à Reims dans une imprimerie spécialement réquisitionnée à cet effet $[. .$.

$2^{\circ}$ - Les autorités militaires britanniques et françaises prendront les dispositions nécessaires pour faire transporter les tracts imprimés à Reims directement de cette ville aux parcs d'aviation ou d'aérostation désignées par elles.

$3^{\circ}$ - Les tracts [...] seront distribués sur le front indifféremment par des aviateurs anglais ou par des aviateurs français ${ }^{86}$.

Or, les Français montrent au mois de septembre déjà quelques réticences ${ }^{87}$. Tout d'abord, en ce qui concerne l'impression commune à Reims : bien que la réquisition d'une imprimerie en France ait été débattue de manière approfondie de mai à août 1939 entre la Commission de Défense Impériale et le Secrétariat Général du Conseil Supérieur Défense Nationale $^{88}$, ce projet n'aboutit pas. Après une visite du comité franco-britannique de la propagande aux imprimeries de Reims ${ }^{89}$, une longue étude et un devis très détaillé révélent le caractère onéreux de l'entreprise ${ }^{90}$. Les Alliés décident alors le 3 novembre 1939, de part et d'autre, de continuer l'impression de leurs tracts au niveau national ${ }^{91}$. Cette étape n'étant pas accomplie, les aviateurs anglais continuent de distribuer les tracts anglais et les aviateurs français les tracts français. De même, pendant les réunions de l'Entre-deux-Guerres du Comité franco-britannique, l'accent avait été mis sur la nécessité de fabriquer des ballons en

\footnotetext{
${ }^{82}$ AN F 41/980.

${ }^{83}$ AN 72 AJ 582, «Collaboration franco-britannique pour l'action de propagande sur l'ennemi », 12 février 1940.

${ }^{84}$ Sur ce sujet voir l'excellente analyse de Georgakakis Didier, op.cit., 2004.

${ }^{85}$ NA FO 898/194 « Meeting held on September 18th, at 18-20 place de la madeleine, Paris. »

${ }^{86} \mathrm{AN} \mathrm{F} 41 / 980$.

${ }^{87}$ NA FO 898/195.

${ }^{88}$ NA FO 898/ 194-195.

89 AN F 41/991 «Note pour le Commissaire général Comité franco-britannique de la propagande chez l'ennemi », 5 septembre 1939.

${ }^{90}$ AN F 41/991 «Note sur l'installation à Reims d'une imprimerie destinée à la confection des tracts de propagande anglais et français », 13 octobre 1939.

${ }^{91}$ AN F 41/991 « Note pour Monsieur le Ministre de la Défense nationale et de la guerre », 3 novembre 1939
} 
temps de paix pour éviter « une impossibilité de lancer une offensive de propagande ${ }^{92}$ si les avions devaient être mobilisés pour des opérations militaires. Cependant la réalité face à la guerre est différente. En France, aucun ballon anglais ni nouveau ballon français n'a été produit avant le déclenchement de la guerre. Le 11 septembre 1939, dans une lettre adressée à l'Armée de l'Air, la IIIème Division du Commissariat général à l'Information déplore :

La construction des ballons anglais est à l'étude en France. Mais il ne faut pas compter qu'elle se réalise avant un ou deux mois. [...] Les Anglais ne sont pas en état de nous en livrer provenant de leur propre construction $^{93}$.

Une pénurie de ballons modernes sévit à la fois du côté français et du côté anglais. La solution pour les autorités françaises est alors d'utiliser d'anciens modèles de ballons, ceux de la Grande Guerre, en attendant que de nouveaux soient disponibles. C'est ainsi que, pour le premier mois de guerre, les Français envoient leurs tracts par le biais de ballons « en papier », qui peuvent «transporter environs 1500 tracts à une quarantaine de kilomètre ${ }^{94}$. À partir du 7 octobre 1939, la production des ballons modernes en caoutchouc français est lancée. Les techniques des Alliés restent néanmoins plus efficaces que celles de leurs ennemis au début de la guerre, ceux-ci utilisant des ballons de baudruches ${ }^{95}$. En que qui concerne l'aviation, il avait été déclaré au sein du Comité franco-britannique de propagande de juin 1939 que «le lancement de tracts est plus économique et plus effectif lorsqu'il est fait par l'Aviation de bombardement ou l'Aviation de reconnaissance» ${ }^{96}$ que par ballons. Cependant, il faut là aussi s'adapter, la guerre étant déclenchée. Parallèlement à la technique rudimentaire de l'envoi des tracts à la main depuis les avions, les Français développent un système de largage des tracts, le « chargeur $32 »^{97}$. Celui-ci est initialement un chargeur pour petite bombe, utilisé ensuite pour répandre entre 192000 et 762000 tracts en fonction du type d'avion, sur les lignes ennemies ${ }^{98}$. Les autorités essaient ainsi de contourner les problèmes techniques, le manque de matériel dû notamment aux retards dans les délais de livraison et aux restrictions budgétaires. Les résolutions prises lors de l'Entre-deux-Guerres se heurtent à la réalité de la guerre, aux limites d'ordre technique, mais également humaines. La coopération très étroite qui avait été prévue au sortir de la Grande Guerre n'est donc que partiellement atteinte.

En comparant les plans initialement prévus de l'Entre-deux-guerres concernant la coopération en matière de propagande vers l'ennemi avec les réalisations concrètes de la « drôle de guerre », un bilan mitigé doit être dressé. Il ne fait aucun doute que l'expérience de la Grande Guerre, et notamment la prise de conscience des autorités militaires et civiles aussi bien françaises que britanniques ont joué un rôle déterminant dans l'élaboration d'une propagande commune pendant l'Entre-deux-Guerres. Ce dialogue régulier se poursuit et s'intensifie avec l'éclatement du second conflit mondial : de nombreux renseignements sur l'Allemagne ainsi que des informations sur les techniques matérielles de diffusion de tracts sont échangées entre les deux Alliés. En outre, des lignes directrices de propagande sont élaborées et un discours commun de propagande pour l'ennemi et visible par l'ennemi, réussi à émerger. Néanmoins, si certains des projets se concrétisent, comme l'élaboration du « tract

\footnotetext{
${ }^{92}$ AN F/41/980 « La valeur des ballons comme moyen de transport de tracts », juin 1939.

${ }^{93}$ AN F/41/991, «Etude du lancement des tracts par ballons », 11.9.1939.

${ }^{94}$ Kirchner, op. cit., p. 34.

95 BArch-MA, RW4/242, «Flugblattabwurf » RMVP an das OKW, 18.11.1939. Dans ce rapport allemand, l'auteur insiste pour ne plus utiliser de « Kinderluftballon » (ballons pour enfants) en expliquant que cela ne fait « certainement pas une bonne impression ».

${ }^{96}$ AN F/41/980 « La valeur des ballons comme moyen de transport de tracts », juin 1939.

${ }^{97}$ Kirchner, op. cit., p. 34.

${ }^{98}$ Ibidem.
} 
commun », d'autres projets sont modifiés, voir même abandonnés : il en va ainsi de la mise en fonction de l'imprimerie de Reims qui échoue faute de dynamisme du côté français. Là où des difficultés persistent, leurs origines ne sont, dans la plupart des cas, pas liées aux divergences de point de vue sur les thèmes à évoquer, mais sont à rattacher aux manques de moyens matériels et financiers et au manque de confiance vis-à-vis de l'autre.

Cependant, la coopération franco-britannique dépasse le domaine de la propagande vers les pays ennemis. Elle est couplée d'une organisation permettant à chacun des deux alliés de promouvoir l'image de l'autre dans son propre pays. Les autorités françaises et anglaises reçoivent par exemple des photos de l'armée alliée transmises ensuite aux journaux nationaux afin de contrecarrer la propagande antibritannique allemande en France. Une étude plus approfondie de cet aspect de la coopération britannique permettrait de parfaire le tableau du travail de propagande réalisé communément entre la France et la Grande-Bretagne.

\section{MAUDE FAGOT}

\section{Résumé :}

Après avoir fait une brève apparition à la fin de la Première Guerre mondiale, la mise en commun des techniques et méthodes des propagandes alliées n'est réellement mise en pratique que lors de la Seconde Guerre mondiale. Le dialogue qui s'instaure entre la France et la Grande-Bretagne est le fruit d'un long travail de préparation pendant l'Entre-deux-guerres. À partir de septembre 1939, les deux alliés initient une coordination de leurs savoirs en matière de propagande, ouvrant ainsi un nouveau domaine de coopération interallié. Celui-ci nécessite une entente non seulement au niveau militaire mais également au niveau idéologique. Dans certains cas, les propagandistes et responsables français et anglais se heurtent à des obstacles d'ordre technique ou d'intérêt national.

Astract :

After making a short appearance at the end of the First World War, the pooling of technic and methods of allied propaganda begin in the practice not before the Second World War. The dialog, which emerged between France and Great Britain, is the fruit of a long preparations work during the interwar period. From the 2d September 1939 both allies initiated a coordination of their knowledge in propaganda, opening a new field of inter-Allied cooperation. This needs a good understanding not only about military issues but also about ideological themes. In certain cases the propagandists and the persons in charge are confronted to obstacles of a technical nature or concerning the national interest. 